\title{
Optimum structured adsorbents for gas separation processes
}

\author{
Fateme Rezaei, Paul Webley* \\ Department of Chemical Engineering, Monash University, VIC 3800, Australia
}

\section{A R T I C L E I N F O}

\section{Article history:}

Received 2 June 2009

Received in revised form 19 August 2009

Accepted 22 August 2009

Available online 1 September 2009

\section{Keywords:}

Structured adsorbent

Mass transfer zone

Pressure drop

Adsorbent loading

\begin{abstract}
A B S T R A C T
Recent developments in separation technology by adsorption have included the development of new structured adsorbents which offer some attractive characteristics compared to a typical packed bed. These improved features include lower energy consumption, higher throughput and superior recovery and purity of product. However, the exact combination of structural, geometric parameters which yields optimum performance is unknown. This study formulates a methodology for comparison based on a variety of analytical and numerical models and uses it to examine the performance of different adsorbent configurations. In particular, monolithic, laminate and foam structures are evaluated and compared to a packed bed of pellets. The effects of physical adsorbent parameters which govern the performance of a PSA process are considered during model development. Comparisons are carried out based on mass transfer kinetics, adsorbent loading and pressure drop of a PSA system for $\mathrm{CO}_{2} / \mathrm{N}_{2}$ separation. The results indicated that structured adsorbents can provide superior throughput to packed beds provided their geometrical parameters exceed certain values. For example, laminate structures can offer superior performance to a packed bed of pellets only if the critical sheet thickness and spacing are less than about $0.2 \mathrm{~mm}$. Each adsorbent structure should be designed to operate at its "optimal" velocity. When operating at velocities higher than the "optimal" value, the increase in pressure drop and length of the mass transfer zone more than offsets gains accrued through reduction in cycle time.
\end{abstract}

(c) 2009 Elsevier Ltd. All rights reserved.

\section{Introduction}

Efficient gas separation processes operating at high throughput are compromised when adsorbent materials in the form of beads or granules are used. High pressure drop associated with gas flow through a packed bed of beads/pellets and mass transfer limitations related to gas diffusion into or out of the beads rapidly reduce system performance. To address these drawbacks, different adsorbent structures may be considered.

Recently, various structured adsorbents with enhanced adsorption characteristics such as monolithic, laminate and foam structures have gained considerable attention as substitutes for traditional adsorbent particles (Kodama et al., 1993; Maurer, 1994; Gadkaree, 1997; Li et al., 1998; Golden et al., 2003; Brandani et al., 2004; Keefer et al., 2004; Sawad et al., 2005; Golden et al., 2005; Zabka et al., 2006; Grande et al., 2006; Rode et al., 2007).

Parallel channel monolithic structures with controllable shape, cell density and wall thickness have been reported for their use in adsorptive gas separation systems (Kodama et al., 1993; Gadkaree, 1997; Li et al., 1998; Lee et al., 2000; Yu et al., 2002; Yates et al.,

\footnotetext{
* Corresponding author. Tel.: +61 39905 3445; fax: +61 399055686

E-mail address: paul.webley@eng.monash.edu.au (P. Webley).
}

2003). The main advantage of such configurations resides in their low pressure drop and higher mass transfer rates. Although it has been reported that the mass transfer characteristics of monolithic adsorbents is somewhat inferior to a comparable packed bed, this is more a consequence of early monolithic structures with thick walls rather than any intrinsic limitation. It would be possible to improve the mass transfer behaviour of monoliths by utilizing structures with reduced channel width and wall thickness or more appropriate shape. Patton et al. (2004) suggests the employment of hexagonal channels rather than ordinary square or circular channels. These authors applied the linear driving force model (LDF) to optimize practical monolith channels taking mass transfer and pressure drop characteristics into account. In recent study performed by Zabka et al. (2008), the performances of the separation of chiral species by the simulated moving bed (SMB) unit packed with conventional adsorbent beads and monolithic adsorbents were compared. The authors concluded that the selection of particle diameter or different bed morphologies is a trade-off between the productivity and eluent consumption.

The use of parallel laminate structures for adsorption processes is rather new with a few patents describing the use of adsorbent materials in the form of laminate sheets in adsorptive gas separation applications. Rode et al., 2007 demonstrated the application of improved adsorbent sheet based parallel passage structures for PSA, TSA and partial PSA devices. In order for laminates to be effective 
adsorbents, the thickness of the sheets and the space between adjacent sheets must be as small as possible but not so small so as to result in excessive pressure drop. Ruthven and Thaeron (1996) carried out an experimental and theoretical study on the performance of a parallel passage contactor based on HETP (height equivalent to a theoretical plate) and pressure drop. Their results emphasize the advantage of such an adsorber over the conventional beads for applications in which pressure drop is a key factor.

Ceramic foams have many attractive features as catalyst supports; however, their utilization in adsorptive gas separation applications is scarce. Open cell foams comprising a network of solid struts and pores offer substantial benefits over configurations comprising external supports; namely, a low pressure drop operation with tortuous flow path and high geometric surface area which enhance the rate of external mass transfer (Richardson et al., 2003; Patcas et al., 2007).

Recently, Patcas et al. (2007), performed an experimental comparison of catalysts with different carriers, namely, honeycomb monoliths, ceramic foams and spherical particles based on their pressure drop and mass and heat transfer characteristics in the oxidation of carbon monoxide. Their results showed that the performance of foam catalysts with respect to combined high mass transfer and low pressure drop were superior over particles and inferior to that of honeycomb structures.

In our recent review of the use of structured adsorbents (Rezaei and Webley, 2009), we identified the important structural parameters that impact the overall performance of gas separation processes. These are: (1) amount of adsorbent contained in a given volume (adsorbent loading), (2) pressure drop through the structure per unit length, (3) external surface area per unit volume, (4) total void volume and (5) channelling and dispersion parameter characteristics. Some of these parameters are constants of the zeolite structure (1), (3), (4) while others also depend on gas velocity (2), (5).

As is well established, for cyclic processes such as PSA or TSA, reducing cycle time is the most important factor to improving the process in terms of reducing adsorbent inventory and cost since shorter cycles result in smaller beds. However, product recovery often decreases as cycle time is decreased unless adsorption rate is correspondingly increased (Ackley et al., 2003). In order to capture this feature, the well known mass transfer zone (MTZ) concept (also referred to as length of unused bed or LUB) was used here to assess the performance of a range of adsorbent structures.

It is clear that the parameters of a particular adsorbent structure will influence the separation performance and cost. It is also evident that a trade-off will exist between mass transfer, pressure drop and adsorbent loading as the geometry of the adsorbent structures are altered. It follows that for each structure there is an optimum set of geometric parameters which will provide the "best" performance as defined by a particular objective function. It is the goal of this study to develop the analytical framework required to determine this optimum set of geometric parameters and to use this model to examine laminate, monolith and foam structures.

\section{Model description}

\subsection{Mass transfer models}

A number of simplified mathematical models were developed for different geometries to describe the adsorption of a single component in an inert carrier. A differential mass balance for the adsorbate in the gas phase flowing through a structure (independent of geometry) gives

$\varepsilon_{b} \frac{\partial C}{\partial t}-D_{L} \frac{\partial^{2} C}{\partial z^{2}}+\frac{\partial U C}{\partial z}=-\bar{k} a\left(C-\bar{C}^{p}\right)$
Table 1

Adsorption parameters related to each structured adsorbent.

\begin{tabular}{lllll}
\hline Adsorbent & $\bar{k}$ & $a$ & $\alpha$ & $\varepsilon_{b}$ \\
\hline Pellet/bead & $\frac{1}{\frac{1}{k_{f}}+\frac{R_{p}}{5 D_{e}}}$ & $\frac{3\left(1-\varepsilon_{b}\right)}{R_{p}}$ & $\frac{3}{R_{p}}$ & $0.35-0.4$ \\
Monolith & $\frac{1}{\frac{1}{k_{f}}+\frac{R_{2}^{2}-R_{1}^{2}}{4 R_{1} D_{e}}}$ & $\frac{2 \varepsilon_{b}}{R_{1}}$ & $\frac{2 R}{R_{2}^{2}-R_{1}^{2}}$ & $\left(\frac{2 R_{1}}{R_{1}+R_{2}}\right)^{2}$ \\
Laminate & $\frac{1}{\frac{1}{k_{f}}+\frac{W}{6 D_{e}}}$ & $\frac{2 \varepsilon_{b}}{d}$ & $\frac{2}{W}$ & $\left(\frac{d}{d+2 W}\right)$ \\
Foam & $\frac{1}{1}+\frac{R_{p}}{4 D_{e}}$ & $\frac{2 \varepsilon_{b}}{R_{p}}$ & $\frac{2}{R_{p}}$ & $0.75-0.85$ \\
\hline
\end{tabular}

where $C$ and $\bar{C}^{p}$ are the concentration of the adsorbate in the gas phase and the average concentration in the pores of the adsorbent, respectively, $a$ is specific surface area per unit bed volume; $U$ is the superficial fluid velocity; $\bar{k}$ is the overall (or total) mass transfer coefficient; $D_{L}$ is the axial dispersion coefficient and $\varepsilon_{b}$ is the bed void fraction. As written, Eq. (1) includes axial dispersion explicitly. A differential mass balance for the adsorbate inside the solid phase is given by

$\varepsilon_{p} \frac{\partial \bar{C}^{p}}{\partial t}+\rho_{s} \frac{\partial \bar{q}}{\partial t}=+\bar{k} \alpha\left(C-\bar{C}^{p}\right)$

where $\varepsilon_{p}$ is the solid void fraction; $\alpha$ is the geometric factor which is different for each adsorbent structure; $\rho_{s}$ is the adsorbent density (includes binder, support, etc.) and $\bar{q}$ is the average adsorbent loading. The initial and boundary conditions appropriate to a breakthrough simulation for the above equations are

$C(t=0)=0, \quad \bar{q}(t=0)=0, \quad \bar{C}^{p}(t=0)=0$

$C_{0}+\left.\frac{\partial C}{\partial z}\right|_{z=0}=0$

$\left.\frac{\partial C}{\partial z}\right|_{z=L}=0$

The boundary condition at the inlet of the column is given by Danckwert's boundary condition (Eq. (4)). This entry condition matches the combined advective and dispersive concentration flux. However, after running models, it was found that the term $\left.\frac{\partial C}{\partial z}\right|_{z=0}$ is negligible and the inlet boundary condition can be simplified to

$C(z=0)=C_{0}$

A detailed description of the model development for monoliths is included in Appendix A. Eqs. (1) and (2) are based on the assumption that the concentration profile in the solid phase is parabolic. The constants in Eqs. (1) and (2) related to each structure have been summarized in Table 1. In this model, local equilibrium is assumed to exist between the adsorbed and the gas species within the pores (i.e. there is only pore resistance in the system). It is worth mentioning here that the monolith is represented by a series of parallel cylinders, however, for the sake of simplicity we assume the same behaviour for all the cylinders (no maldistribution), and that the monolith can be described by a single channel (Young and Finlayson, 1974). Foams are characterized in terms of a pore diameter, $d_{p}$, or pore radius, $R_{p}$, which corresponds to the average size of interconnecting windows and correlates well with the pore density (pores per inch or PPI). In Fig. 1 the geometrical parameters of monoliths and laminates are shown. $R_{1}$ and $R_{2}$ are the inner and outer wall radii of the monolith channel, respectively, and $d$ and $w$ are the laminate gap and wall thickness, respectively. 

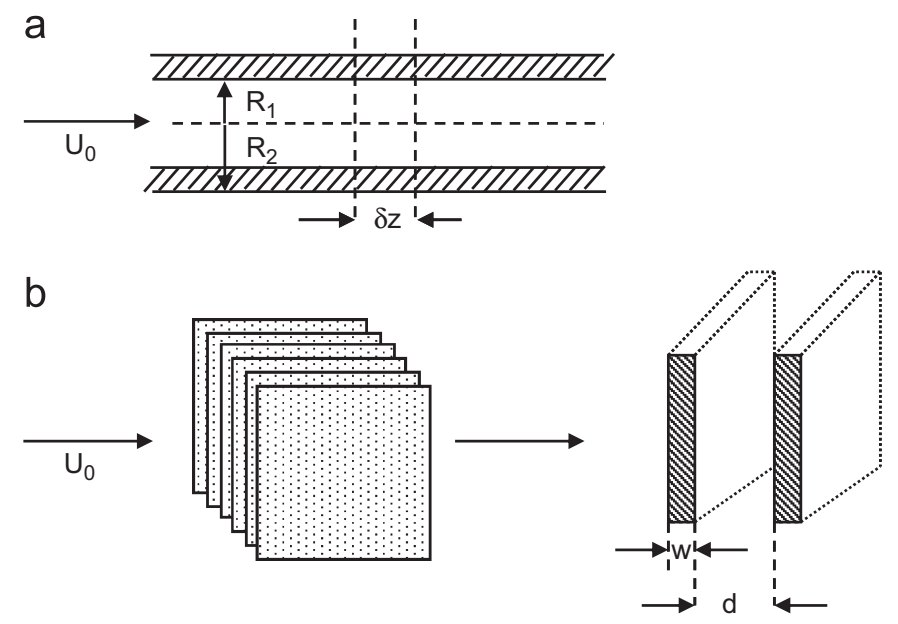

Fig. 1. Structured adsorbents: (a) monolith and (b) laminate.

The total mass transfer coefficient can be represented as follows:

$\frac{1}{\bar{k}}=\frac{1}{k_{f}}+\frac{1}{k_{p}}$

where $k_{f}$ and $k_{p}$ are film and pore mass transfer coefficients, respectively. The pore mass transfer coefficient, $k_{p}$, for pellets, monoliths, foam and laminate structures are

$$
\begin{aligned}
& \left(k_{p}\right)_{\text {pellets }}=\frac{5 D_{e}}{R} \\
& \left(k_{p}\right)_{\text {monoliths }}=\frac{4 D_{e} R_{1}}{R_{2}^{2}-R_{1}^{2}} \\
& \left(k_{p}\right)_{\text {foams }}=\frac{4 D_{e}}{R} \\
& \left(k_{p}\right)_{\text {laminates }}=\frac{6 D_{e}}{w}
\end{aligned}
$$

where $D_{e}$ is the effective diffusivity which is taken to be identical for different structures, since we assume here that the intrinsic pore structure of the adsorbent is the same. This latter constraint is not required but was adopted here for simplicity in the comparison of structures. Indeed, some advantages of novel adsorbent structures may well be that superior $D_{e}$ can be achieved by engineering the pore structure into the adsorbent structure. The solid adsorbent is assumed to have the same properties in all adsorbent structures.

The appropriate dimensionless group characterising film diffusion is the Sherwood number, Sh. For pelleted systems the Wakao and Funazkri (1978) equation was used to estimate $k_{f}$

$S h=2+1.1 R e^{0.6} S c^{0.33}$

For monolithic and laminate structures, Hawthorn (1974) equation for laminar flow was employed

$S h=\frac{d k_{f}}{D_{m}}=A\left(1+0.139 \operatorname{ReSc} \frac{d}{L}\right)^{0.81}$

where the parameter $A$ is 3.6 for circular channels, 2.35 for triangular channels and 2.95 for square channels. $D_{m}$ and $L$ are molecular diffusion coefficients and column length, respectively.

A generalized correlation proposed by Groppi et al. (2007) was employed to describe the external film mass transfer coefficient in foam structures

$S h=0.91 R e^{0.43} S c^{0.33}$
Table 2

Film and pore mass transfer expressions for structured adsorbents.

\begin{tabular}{lll}
\hline Adsorbent & $k_{f}$ expression & $k_{p}$ expression \\
\hline Pellet/bead & $S h=2+1.1 \operatorname{Re}^{0.6} S c^{0.33}$ & $k_{p}=\frac{5 D_{e}}{R}$ \\
Monolith & $S h=2.696\left[1+0.139\left(\frac{2 R_{1}}{L}\right) R e S c\right]^{0.81}$ & $k_{p}=\frac{4 D_{e} R_{1}}{R_{2}^{2}-R_{1}^{2}}$ \\
Laminate & $S h=2.696\left[1+0.139\left(\frac{d}{L}\right) R e S c\right]^{0.81}$ & $k_{p}=\frac{6 D_{e}}{w}$ \\
Foam & $S h=0.91 R e^{0.43} S c^{0.33}$ & $k_{p}=\frac{4 D_{e}}{R}$ \\
\hline
\end{tabular}

subject to the constraint

$15<R e<200$

A summary of $k_{f}$ and $k_{p}$ expressions related to each configuration is presented in Table 2 .

Since axial dispersion acts to disperse the breakthrough front in an adsorbent system, it is important to include its effect. Axial dispersion in a packed bed is correlated in terms of the Peclet number $\left(P e=U d / D_{L}\right)$, and can be obtained from

$\frac{\varepsilon_{b} D_{L}}{D_{m}}=20+0.5 \operatorname{ReSc}$

where $D_{m}$ is the molecular diffusion coefficient. The same expression was used for foam structures. In laminar flow, axial dispersion in monolithic structures can be calculated from Taylor (1954) analysis

$D_{L}=D_{m}+\frac{1}{192} \frac{U^{2}\left(2 R_{1}\right)^{2}}{D_{m}}$

For laminates, a similar approach holds as with monoliths.

The inclusion of axial dispersion can be done either through the term $D_{L} \partial^{2} C / \partial z^{2}$ or, since its effect is to disperse the mass transfer front, it can be represented through the mass transfer coefficient, $k$. In our numerical study presented later, it was included explicitly through the term $D_{L} \partial^{2} C / \partial z^{2}$. However, for linear adsorption isotherm models, the contribution of dispersion can alternatively be estimated by adding the term $D_{L} / U^{2}$ to the mass transfer coefficient of the adsorbent (Ruthven, 1984), thus the overall rate constant is

$\left(\frac{1}{k}\right)_{\text {overall }}=\left(\frac{1}{k}\right)_{\text {mass_transfer }}+\frac{D_{L}}{U^{2}}$

Estimation of mass transfer rate constant, $k_{\text {mass_transfer }}$ is performed by assuming a parabolic concentration profile in the adsorbent material (as discussed earlier and derived in the appendix) and a linear driving force based on a gas concentration driving force model. The mass transfer coefficient $k_{\text {mass_transfer }}$ for different structures is

Pellets : $\left(\frac{1}{k}\right)_{\text {mass_transfer }}=\frac{\varepsilon_{b}}{1-\varepsilon_{b}}\left(\frac{R_{p}}{3 k_{f}}+\frac{R_{p}^{2}}{15 D_{e}}\right)$

Monolith : $\left(\frac{1}{k}\right)_{\text {mass_transfer }}=\frac{R_{1}}{2 k_{f}}+\frac{R_{2}^{2}-R_{1}^{2}}{8 D_{e}}$

Laminate : $\left(\frac{1}{k}\right)_{\text {mass_transfer }}=\frac{d}{2 k_{f}}+\frac{W d}{6 D_{e}}$

Foam : $\left(\frac{1}{k}\right)_{\text {mass_transfer }}=\frac{R_{p}}{2 k_{f}}+\frac{R_{p}^{2}}{8 D_{e}}$ 


\subsection{Pressure drop}

The pressure drop of the adsorbent structures was calculated using well known equations for each case. For pellets, the Ergun (1952) equation was used

$$
\frac{\Delta P}{L}=150 \frac{(1-\varepsilon)^{2}}{\varepsilon^{3}} \frac{\mu}{d_{p}^{2}} U+1.75 \frac{(1-\varepsilon)}{\varepsilon^{3}} \frac{\rho}{d_{p}} U^{2}
$$

where, $\rho$ and $\mu$ are density and viscosity of the flowing gas. For monolithic and laminate structures, the Hagen-Poiseuille equation (Cybulski and Moulijn, 1998) was applied

$$
\frac{\Delta P}{L}=\frac{32 \mu}{d_{p}^{2} \varepsilon_{b}} U
$$

Although the Ergun equation has often been used to describe the pressure drop of open-cell foams, in this work the pressure drop in the foam adsorbents was calculated from equations recently proposed by Richardson et al. (2000) in which the authors, based on the work of Gibson and Ashby (1997) considered that the cellular medium is formed by uniform tri-dimensional cells.

$\frac{\Delta P}{L}=\frac{\alpha_{1} s^{2}(1-\varepsilon)^{2}}{\varepsilon^{3}} \mu U+\frac{\alpha_{2} s(1-\varepsilon)}{\varepsilon^{3}} \rho U^{2}$

where $\alpha_{1}$ and $\alpha_{2}$ and $s$ can be calculated using following expressions:

$$
\begin{aligned}
& \alpha_{1}=973 d_{p}^{0.743}(1-\varepsilon)^{-0.0982} \\
& \alpha_{2}=368 d_{p}^{-0.7523}(1-\varepsilon)^{0.07158} \\
& s=\frac{12.979\left[1-0.971(1-\varepsilon)^{0.5}\right]}{d_{p}(1-\varepsilon)^{0.5}}
\end{aligned}
$$

\subsection{Characterisation and calculation of the mass transfer zone (MTZ)}

Adsorbent systems with non-linear and favourable isotherms exhibit constant pattern behaviour during adsorption (Sircar et al., 1983). By employing such constant pattern conditions, it is possible to evaluate the mass transfer zone characteristics of the system. In this work, the MTZ is created by dispersion resulting from all of the dispersive forces in the system as encapsulated by the overall (total) rate constant $k$. The width of the mass transfer zone is the product of the zone velocity and the extent of spreading

$M T Z=\beta\left(t_{2}-t_{1}\right)$

where $t_{2}$ and $t_{1}$ are the two times of breakthrough (5\% and $95 \%$ of the outlet concentration). The zone velocity, $\beta$, can be obtained from a simple rearrangement of the governing equations. If we add the balance equations for the solid and gas phases, we obtain the single equation

$\varepsilon_{b} \frac{\partial C}{\partial t}+\frac{\partial U C_{i}}{\partial z}+\left(\frac{a}{\alpha}\right) \rho_{s} \frac{\partial \bar{q}}{\partial t}=0$

This balance equation is obtained based on these assumptions that the accumulation in the particle pores is neglected and axial dispersion term is also neglected. Assuming constant velocity, the velocity of the mass transfer zone, $\beta$ is therefore approximately by

$$
\beta=\frac{U}{\varepsilon_{b}+\left(\frac{a}{\alpha}\right) \rho_{s} \frac{q_{\text {feed }}}{c_{\text {feed }}}}
$$

The isotherm slope therefore appears in the expression for the MTZ, as expected. The difference in breakthrough time $t_{1}$ and $t_{2}$ can be derived from constant pattern analyses as shown by Sircar et al. (1983) and it follows that $t_{2}-t_{1}$ is inversely proportional to the overall rate constant, $\bar{k}$, for constant pattern conditions. Thus $\left(t_{2}-t_{1}\right) \propto 1 /(\alpha \bar{k})$. Combining these equations gives proportionality for the mass transfer zone

$M T Z \propto \frac{U}{\alpha \varepsilon_{b} \bar{k}+a \bar{k} \rho_{s}\left(q_{\text {feed }} / c_{\text {feed }}\right)}$

This equation is simple enough to be implemented in a spreadsheet and shows the major dependency of the MTZ on superficial velocity, $U$, and rate constant as well as geometric factors $a$ and $\alpha$. It should be noted that linear systems do not show constant pattern behaviour in the presence of dispersive phenomena such as mass transfer resistance and axial dispersion although qualitatively Eq. (30) will indicate the expected trends. Although (30) is useful to provide a relative assessment of the impact of variables on MTZ (and is used later to derive a heuristic model), it is possible and desirable to read off the length of the mass transfer directly from the numerical simulations in the $z$-domain. This was done as described below.

\subsection{Numerical solution of the conservation equations and basis for simulation}

Simulations of the numerical models for different adsorbent geometries were performed using COMSOL 3.5a. The conservation equations were solved using the Lagrange quadratic finite element method, with a total number of 2424 elements. To confirm that numerical dispersion was not affecting the results, the simulations were routinely tested with more elements (double) with no change in results. The simulations of breakthrough curves in each case do not take more than $20 \mathrm{~s}$. The physical characteristics of structures considered for comparison are presented in Table 3. A cylindrical bed size of $2 \mathrm{~cm}$ diameter and $30 \mathrm{~cm}$ length was chosen arbitrarily. The adsorbent voidage in each case was taken to be the same (0.6) and the amount of binder was taken to be the same ( $10 \%$ by weight). The density of the foam, monolith wall and laminate sheet was taken to be the same as a pellet density. This may not be true of an actual laminate or monolith especially if a support structure is needed. For the sake of comparison, there should be the same amount of adsorbent mass per unit volume of bed for all configurations, while as can be seen from Table 3, the adsorbent mass is different for all geometries. Therefore, in order to compensate the lower amount of active adsorbent in laminates, foams and monolithic configurations, and ensure the same stoichiometric breakthrough time (to allow comparison of breakthrough fronts), the superficial velocity was adjusted for each adsorbent structure.

The gas system used for calculations was $10 \% \mathrm{CO}_{2}$ in $\mathrm{N}_{2}$ and the adsorbent was taken to be $\mathrm{NaX}$. The equilibrium data for $\mathrm{CO}_{2}$ on $\mathrm{NaX}$ at $298.15 \mathrm{~K}$ were taken from Hyun and Danner (1982). Although the actual isotherm is non-linear, the simulation we performed was based on a linear isotherm with the equilibrium constant, $K$, taken from the $\mathrm{CO}_{2}$ adsorption isotherm at lower pressures (up to $10 \mathrm{kPa}$ ). A linear constant of $41 \mathrm{~g} \mathrm{~mol} / \mathrm{kg} \mathrm{kPa}$ was used. It was also assumed that the functionality of the effective diffusion coefficient, $D_{e}$, can be evaluated from the equivalent molecular diffusion coefficient scaled by a voidage-tortuosity term in the molecular diffusion regime in which macropore diffusion is rate-limiting step.

\section{Results and discussion}

\subsection{External surface area comparison}

One of the most important parameters which govern the performance of an adsorbent is its external surface area per unit volume, since the mass transfer rate is directly proportional to this parameter. In Fig. 2, the comparison between the external surface areas 
Table 3

Physical properties of structures used in the computations.

\begin{tabular}{|c|c|c|c|c|c|c|c|c|}
\hline Structure & $\begin{array}{l}\text { Bed } \\
\text { voidage }\end{array}$ & $\begin{array}{l}\text { Adsorbent } \\
\text { mass (g) }\end{array}$ & $\begin{array}{l}\text { Bed density } \\
\left(\mathrm{g} / \mathrm{cm}^{3}\right)\end{array}$ & $\begin{array}{l}\text { Specific surface } \\
\text { area }\left(\mathrm{cm}^{2} / \mathrm{cm}^{3}\right)\end{array}$ & $\begin{array}{l}\text { Adsorbent } \\
\text { width }(\mathrm{mm})\end{array}$ & $\begin{array}{l}\text { Cell size } \\
(\mathrm{mm})\end{array}$ & $\begin{array}{l}\text { Total } \\
\text { voidage }\end{array}$ & $\begin{array}{l}\text { Superficial } \\
\text { velocity }(\mathrm{m} / \mathrm{s})\end{array}$ \\
\hline $0.7 \mathrm{~mm}$ pellet & 0.37 & 64.69 & 0.686 & 54.0 & 0.35 & - & 0.75 & 1 \\
\hline Monolith, $200 \mathrm{cpsi}$ & 0.65 & 35.93 & 0.381 & 18.0 & 0.35 & 1.45 & 0.86 & 0.444 \\
\hline Monolith, $400 \mathrm{cpsi}$ & 0.65 & 35.93 & 0.381 & 25.4 & 0.25 & 1.02 & 0.86 & 0.444 \\
\hline Monolith, $600 \mathrm{cpsi}$ & 0.65 & 35.93 & 0.381 & 31.1 & 0.20 & 0.84 & 0.86 & 0.444 \\
\hline Laminate, $0.5 \mathrm{~mm} \times 0.5 \mathrm{~mm}$ & 0.50 & 51.30 & 0.544 & 20.0 & 0.50 & 0.50 & 0.80 & 0.6344 \\
\hline Laminate, $0.3 \mathrm{~mm} \times 0.3 \mathrm{~mm}$ & 0.50 & 51.30 & 0.544 & 33.3 & 0.30 & 0.30 & 0.80 & 0.6344 \\
\hline Laminate, $0.2 \mathrm{~mm} \times 0.2 \mathrm{~mm}$ & 0.50 & 51.30 & 0.544 & 50.0 & 0.20 & 0.20 & 0.80 & 0.6344 \\
\hline Laminate, $0.1 \mathrm{~mm} \times 0.1 \mathrm{~mm}$ & 0.50 & 51.30 & 0.544 & 100.0 & 0.10 & 0.10 & 0.80 & 0.6344 \\
\hline Foam, 20 PPI & 0.80 & 20.54 & 0.218 & 19.75 & 0.81 & - & 0.804 & 0.318 \\
\hline Foam, 30 PPI & 0.80 & 20.54 & 0.218 & 26.66 & 0.60 & - & 0.816 & 0.318 \\
\hline Foam, 45 PPI & 0.80 & 20.54 & 0.218 & 37.20 & 0.43 & - & 0.813 & 0.318 \\
\hline
\end{tabular}

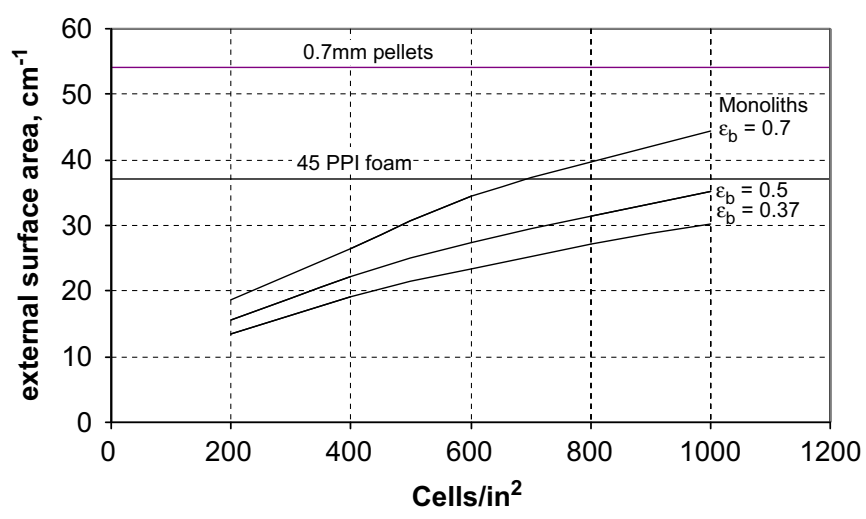

Fig. 2. Comparison of external surface areas for pellet, foam and several monoliths.

per unit volume of $1 \mathrm{~mm}$ bead diameter, 45 PPI foam and different voidage monolithic structures is shown. It is clear that small beaded packed beds and foam adsorbents have very high external surface area which is difficult to match with monolithic structures.

For comparison, a 45 PPI foam and $0.7 \mathrm{~mm}$ pellet diameter packed bed have an external area of 37 and $54 \mathrm{~cm}^{2} / \mathrm{cm}^{3}$, respectively. Monoliths can only match the external surface area by using very high cell densities and high voidages. Unfortunately, high voidage compromises system performance and reduces overall density which more than offsets the gain in external surface area. Practical cell densities of $400 \mathrm{cpsi}$ and voidages of 0.5 will give about $22 \mathrm{~cm}^{2} / \mathrm{cm}^{3}$, a little over half of the surface area of a $1 \mathrm{~mm}$ packed bed. To compensate the smaller surface area of monolithic adsorbents, a thin layer of active film with desirable voidage may be grown on the wall surface. The presence of such a film does not obstruct the gas flow through the channel (due to the small thickness in the range of several microns) while it could enhance the cell density of the active adsorbent.

Fig. 3 shows that the surface area per unit volume of laminate systems compares favourably with packed beds for small laminate spacings and wall thickness. However, practical difficulties associated with maintaining small spacing may complicate deployment of these systems. Furthermore, lower adsorbent loading associated with thinner walls will result in insufficient overall adsorbent density.

\subsection{Comparison of mass transfer characteristics}

The typical mass transfer characteristics of adsorbent structures were calculated using equations presented in Section 2.1 and

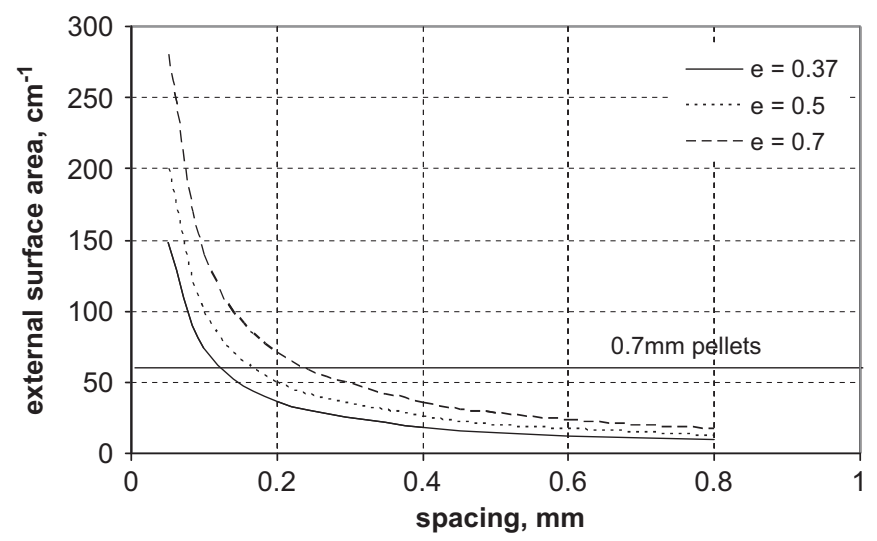

Fig. 3. Comparison of external surface areas for packed bed and several laminates with varying voidages.

the values at a constant superficial velocity of $1 \mathrm{~m} / \mathrm{s}$ are given in Table 4. It is clear from this table that although kinetic properties of beads are somewhat superior to other structures, increasing cell density in monolithic adsorbents, decreasing the spacing between adjacent sheets in laminate configurations or choosing foams with higher number of pore per inch (PPI), can give proportionally improved performance.

Laminate structures can exhibit significantly better performance than a packed bed ( $>50 \%$ ) but only if the critical sheet thickness and spacing is less than about $0.2 \mathrm{~mm}$. As stated earlier, the practical difficulties involved in reproducibly making such structures are not trivial. The tolerances must be quite tight $(<5 \%)$ in the channels to avoid maldistribution. This requires that the spacing must be maintained to within $<0.01 \mathrm{~mm}$. Typically, the mass transfer coefficient of foams would increase by increasing the pore count at constant hydrodynamic conditions due to enhanced surface area available for adsorption. The axial dispersion coefficient of foams is much smaller than dispersion resulting from pore diffusion coefficients and could be justifiably neglected. For example, for 30 PPI foam with characteristic diameter of $0.695 \mathrm{~mm}, D_{L} / U^{2}$ is about $6.4 \times 10^{-4}$.

The influence of monolith external voidage on performance is subtle. At high voidages ( $>0.7$ ), cell walls are thin, high external surface area per unit volume is obtained leading to good mass transfer, however, bed densities are low. These effects counteract each other. The opposite situation occurs at low voidage. The optimal voidage 
Table 4

Mass transfer properties of different adsorbent structures.

\begin{tabular}{|c|c|c|c|c|c|c|c|c|}
\hline Structure & Sc & $\operatorname{Re}$ & Sh & $k_{f}(\mathrm{~cm} / \mathrm{s})$ & $k_{p}(\mathrm{~cm} / \mathrm{s})$ & $U^{2} / D_{L}\left(\mathrm{~s}^{-1}\right)$ & $k_{\text {mass_transfer }}\left(\mathrm{s}^{-1}\right)$ & $k_{\text {overall }}\left(\mathrm{s}^{-1}\right)$ \\
\hline $0.7 \mathrm{~mm}$ pellet & 0.51 & 83 & 14.50 & 34.3 & 4.74 & 543 & 608.00 & 287.00 \\
\hline Monolith, 200 cpsi & 0.51 & 172 & 2.82 & 3.23 & 1.53 & 1480 & 40.7 & 39.61 \\
\hline Monolith, $400 \mathrm{cpsi}$ & 0.51 & 121 & 2.76 & 4.49 & 2.13 & 2920 & 80.63 & 78.51 \\
\hline Monolith, 600 cpsi & 0.51 & 100 & 2.74 & 5.41 & 2.68 & 4200 & 120.49 & 117.13 \\
\hline Laminate, $0.5 \mathrm{~mm} \times 0.5 \mathrm{~mm}$ & 0.51 & 59 & 2.70 & 9.00 & 3.98 & 10500 & 65.24 & 65.24 \\
\hline Laminate, $0.3 \mathrm{~mm} \times 0.3 \mathrm{~mm}$ & 0.51 & 36 & 2.70 & 14.95 & 6.64 & 22300 & 181.11 & 181.11 \\
\hline Laminate, $0.2 \mathrm{~mm} \times 0.2 \mathrm{~mm}$ & 0.51 & 24 & 2.70 & 22.4 & 9.96 & 34300 & 407.41 & 407.41 \\
\hline Laminate, $0.1 \mathrm{~mm} \times 0.1 \mathrm{~mm}$ & 0.51 & 12 & 2.70 & 44.76 & 19.92 & 50700 & 1629.45 & 1629.45 \\
\hline Foam, $20 \mathrm{PPI}$ & 0.51 & 50 & 4.72 & 6.59 & 2.23 & 1380 & 56.04 & 53.86 \\
\hline Foam, 30 PPI & 0.51 & 36 & 4.09 & 9.76 & 3.82 & 1560 & 156.71 & 143.49 \\
\hline Foam, 45 PPI & 0.51 & 24 & 3.43 & 8.38 & 3.91 & 1740 & 158.06 & 143.74 \\
\hline
\end{tabular}

can only be determined once a good idea of the effective diffusion coefficient in the monolith walls is known. Axial dispersion in packed beds ranges from 0 to $2 \mathrm{~cm}^{2} / \mathrm{s}$ at Reynolds numbers greater than 100 which covers most of our region of interest. The axial dispersion contribution to overall mass transfer coefficient for pellets is usually very small (similar to external diffusion) and can justifiably be neglected. Note, however, that very small particles $(<1.0 \mathrm{~mm})$ have significantly more axial dispersion. For example, the limiting Peclet number for $0.7 \mathrm{~mm}$ diameter pellets is about 0.4 giving $D_{L}=18.4 \mathrm{~cm}^{2} / \mathrm{s}$ and $D_{L} / U^{2}=0.0018 \mathrm{~s}$. This is comparable to pore diffusion resistance and indicates that for small diameter pellets, gains in kinetic effects from reducing particle size can be rapidly offset by increased axial dispersion. In monoliths, the axial dispersion is much more dependent on channel diameter and flow rate. In laminar flow, the axial dispersion can be calculated from Taylor's analysis

$P e=\frac{U d}{D_{L}} \approx 192 \frac{D_{m}}{U d}$

This gives $D_{L} / U^{2}=d^{2} /\left(192 D_{m}\right)$. For $1 \mathrm{~mm}$ diameter channel and a molecular diffusivity of $0.1 \mathrm{~cm}^{2} / \mathrm{s}, D_{L} / U^{2}=5 \times 10^{-4} \mathrm{~s}$. This is a negligible contribution to overall dispersion of the mass transfer front. For laminates, a similar conclusion holds as with monoliths. Resistances in the porous material and external film are far greater than axial dispersion in the gas phase.

\subsection{Pressure drop}

The pressure drop at different superficial velocities ranging from 0.1 to $1 \mathrm{~m} / \mathrm{s}$ was calculated using correlations presented at Section 2.2. Fig. 4 shows the pressure drop associated with different adsorbent configurations. As can be seen from this figure, the low density monolith (200 cpsi) exhibited the lowest pressure gradient, while as expected, the highest pressure drop was found to be represented by beads $(0.7 \mathrm{~mm})$. Increasing the monolith cell density to increase adsorbent loading and geometric surface area does not change bed pressure drop as significantly as other adsorbent configurations and the monolithic adsorbents still display the lowest head loss compared to laminate, foam and bead systems. Laminate adsorbents with $0.5 \mathrm{~mm}$ thickness showed a lower pressure drop than foam and beads. According to Giani et al. (2005) and Patcas et al. (2007), the pressure drop characteristics of foam structures are somewhat between those of monoliths and packed beds, our results are in agreement with their findings.

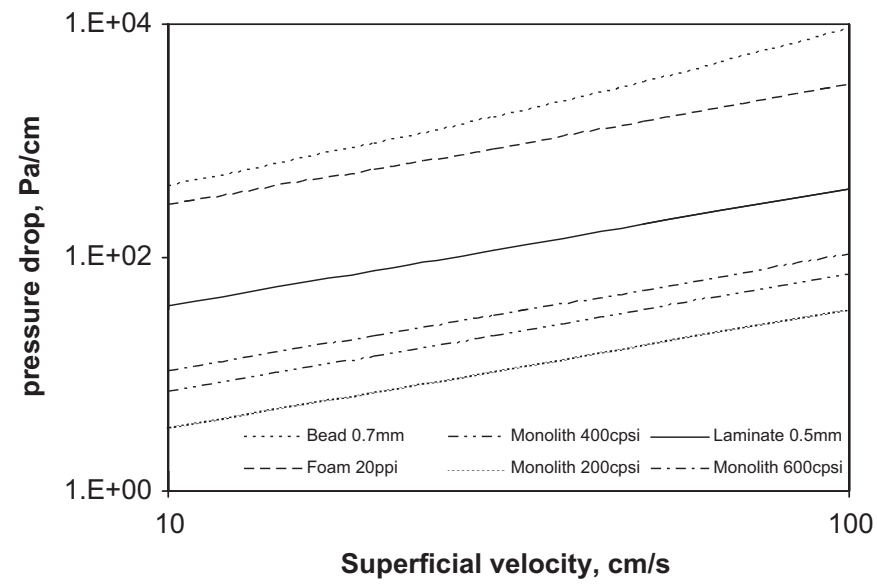

Fig. 4. Comparison of pressure drop for pellet, laminate, foam and several monoliths as a function of superficial gas velocity.

\subsection{Mass transfer zone (MTZ)}

The dynamic behaviour of mass transfer zone was evaluated using different adsorption column operating conditions such as gas flow rates, etc. Fig. 5 shows the mass transfer zone (MTZ) as a function of superficial gas velocity for different adsorbent configurations taken directly from the COMSOL simulations. As expected, for all cases, the mass transfer length was increased by increasing gas velocity. The mass transfer zone length in the case of foam and monolith was considerably longer that of laminate and pellet structures. Laminates with wall thickness of $0.2 \mathrm{~mm}$ show superior mass transfer behaviour to a packed bed of $0.7 \mathrm{~mm}$ beads.

The comparison of $\mathrm{CO}_{2}$ breakthrough curves in different bed configurations is shown in Fig. 6. As can be seen, laminate adsorbents with wall and spacing of $0.2 \mathrm{~mm}$ exhibits a very steep breakthrough front compared to pellets, foams and monoliths. This sharp front is attributed to the much higher value of pore diffusion coefficient, $k_{p}$, in laminate adsorbents as presented in Table 4 . The other structures namely, foam $30 \mathrm{PPI}$, pellet $0.7 \mathrm{~mm}$ and monolith $600 \mathrm{cpsi}$, represent a similar breakthrough front trend, although the times to reach 5\% and $95 \%$ of the outlet concentration are longer in the case of foam adsorbents. As with the same MTZ, the $600 \mathrm{cpsi}$ monolith, 30 PPI foam and $0.2 \times 0.2$ laminate display the same performance. In other words, for adsorptive gas separation processes, using structured adsorbents 


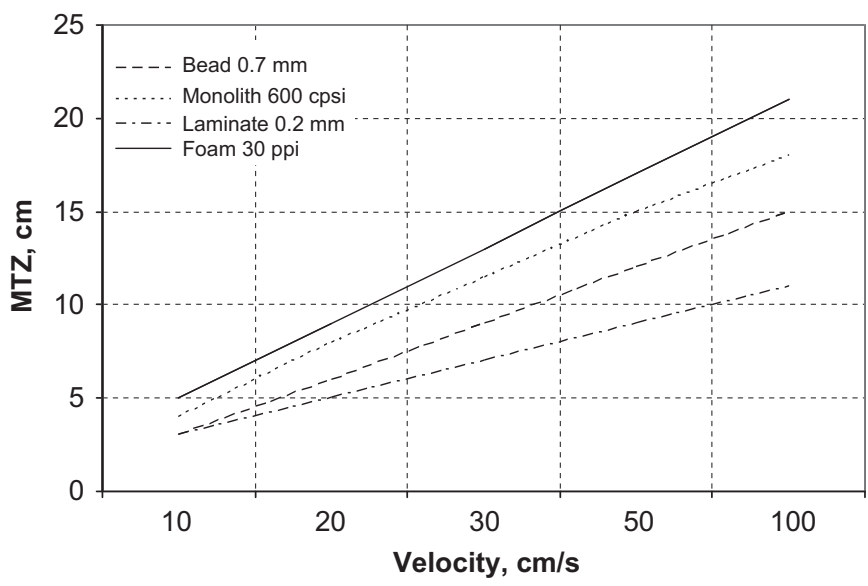

Fig. 5. Comparison of MTZ for pellet, laminate, foam and several monoliths as a function of gas velocity.

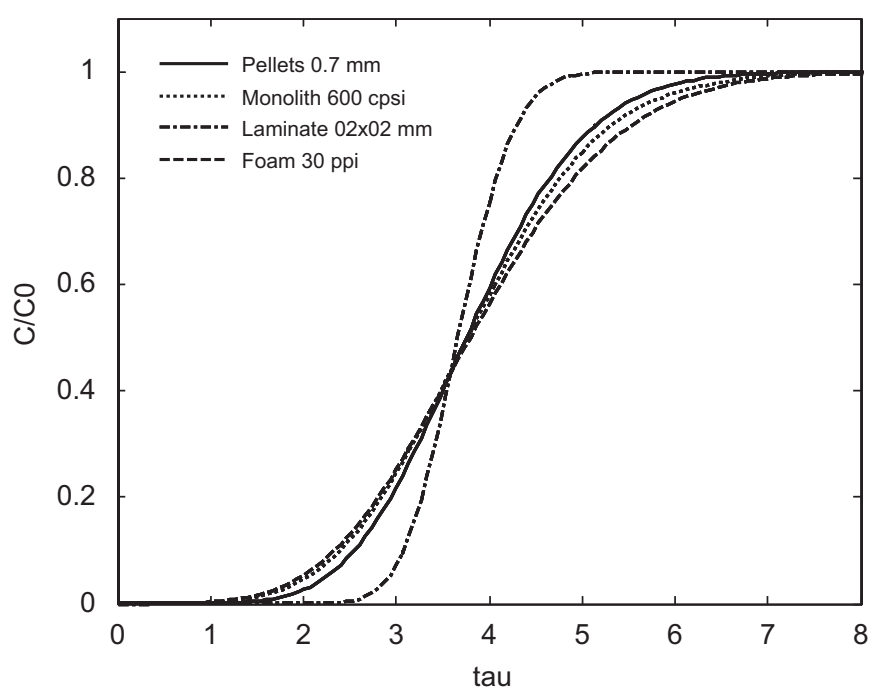

Fig. 6. Comparison of breakthrough curves of $\mathrm{CO}_{2}$ in different bed configurations.

in the form of $600 \mathrm{cpsi}$ monolith, 30 PPI foam and $0.2 \times 0.2$ laminate will give somewhat similar performance to a packed bed of $0.7 \mathrm{~mm}$ diameter beads.

Fig. 7 shows the effect of laminate geometry on breakthrough performance. It is clear that wall thickness and spacing play critical roles in adsorptive performance of laminate structures. Increasing the wall thickness and spacing leads to broadening of breakthrough profiles over a longer time and hence deteriorate the overall system efficiency, while thinner sheets with smaller spacing give sharp breakthrough fronts indicating negligible mass transfer resistance through the bed. A longer MTZ will reduce both working capacities and selectivity of adsorbent.

A similar trend was observed for monolithic structures. Fig. 8 shows that using monoliths with low cell density would give rise to early breakthrough and a longer time until $95 \%$ of outlet concentration is reached. This is due to thicker walls and lower adsorbent mass transfer coefficient $\left(k_{p}\right)$ in the case of lower cell density. Table 4 indicates that axial dispersion in the monolith does not influence the breakthrough front significantly even though axial dispersion increases with increase in cell density.

Breakthrough data for foam adsorbents are shown in Fig. 9. Decreasing pore numbers per inch (PPI) leads to earlier breakthrough

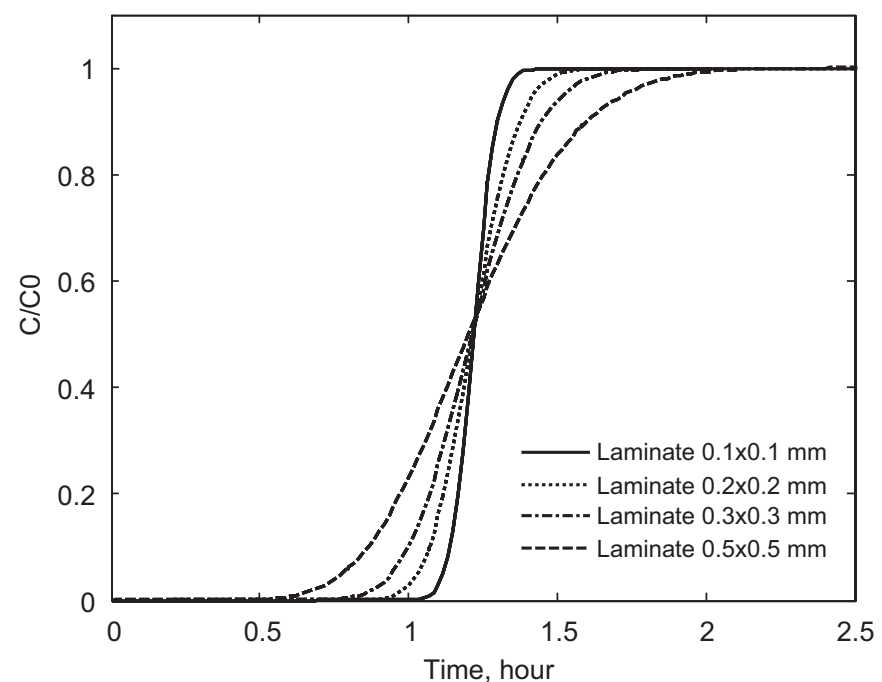

Fig. 7. Comparison of breakthrough curves of $\mathrm{CO}_{2}$ in laminate bed structures with different wall thickness and spacing.

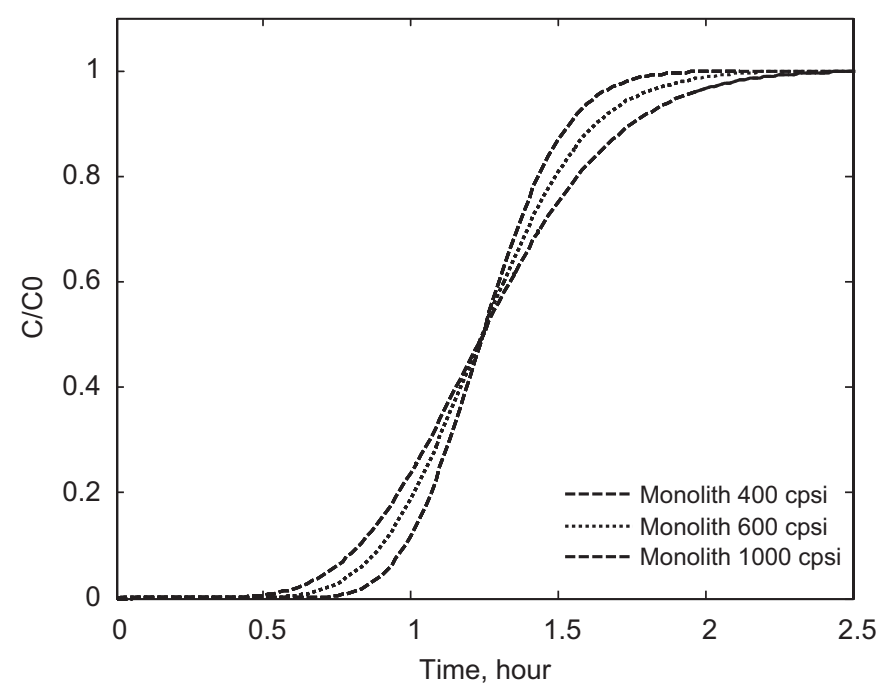

Fig. 8. Comparison of breakthrough curves of $\mathrm{CO}_{2}$ in monolith beds with different cell densities.

and broadening of the front. The effect of axial dispersion on the breakthrough curves of foam adsorbents is clear. By increasing the number of pores (higher PPI) the axial dispersion coefficient is decreased (higher $\left.U^{2} / D_{L}\right)$, however, as evident from Table 4 , the contribution of axial dispersion coefficient on overall rate constant is trivial and hence does not influence the shape of breakthrough front.

\subsection{Effect of adsorbent structure on specific productivity}

The analysis above is useful in identifying features of the adsorbent which impact on pressure drop, mass transfer and adsorbent loading. However, in a process, the goal is usually to maximize specific productivity, i.e. to be able to maximize throughput in a given amount of adsorbent. It is therefore necessary to consider an appropriate combination of process parameters to demonstrate how the pressure drop, cycle time and adsorption front affect system performance. The system specific productivity is the product of the working capacity (moles of gas adsorbed/desorbed per unit mass of adsorbent including binder, support, etc.) and the density of the composite adsorbent. The throughput attainable in a given volume 


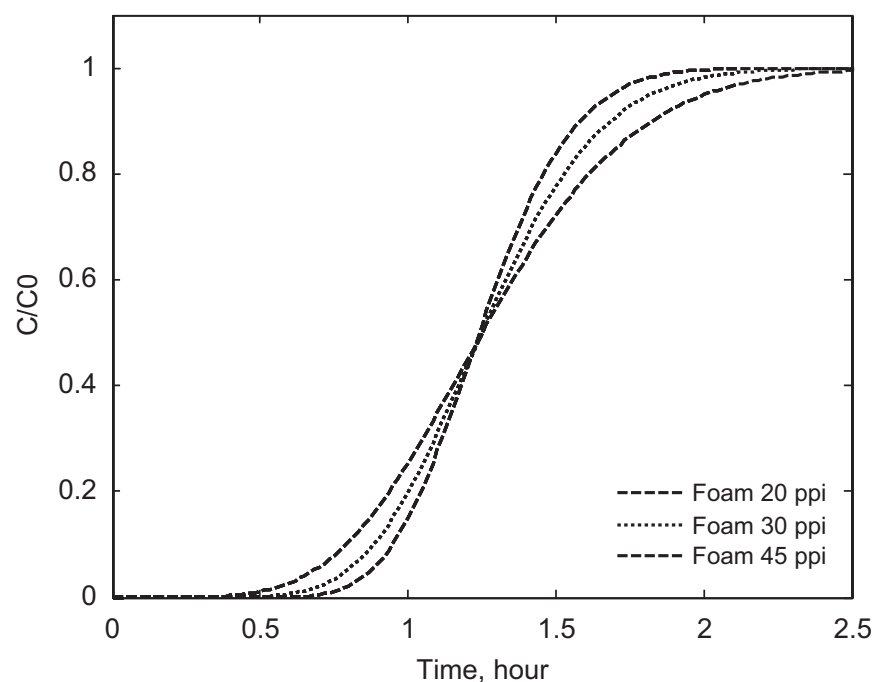

Fig. 9. Comparison of breakthrough curves of $\mathrm{CO}_{2}$ in foam beds with different PPI.

of vessel in a cyclic adsorption system may be expressed as

throughput $(T P D) \propto \frac{\text { gas_production }}{\text { cycle } \times m_{\text {ads }}} \times \frac{\text { cycle }}{\text { day }}$

specific productivity $=k \frac{W C}{\tau}$

where $W C$ is adsorbent working capacity ( $\mathrm{mol} / \mathrm{kg}$ adsorbent/cycle), $m_{a d s}$ is the mass of adsorbent, $\tau$ is the cycle time (s/cycle) and $k$ is a proportionality constant. The effect of pressure drop on the productivity is to reduce the overall working capacity. Instead of operating between a high pressure $P_{H}$ and a low pressure $P_{L}$, the average bed pressure on adsorption is only $P_{H}-\Delta P / 2$ and the bed pressure on desorption is $P_{L}+\Delta P / 2$. Thus the pressure difference between adsorption and desorption is approximately diminished from $P_{H}-P_{L}$ to $P_{H}-P_{L}-\Delta P$. Generally (for any isotherm), the working capacity will be reduced by some function of $\Delta P$ written as $f_{1}(\Delta P)$. The situation for linear isotherms is shown in more detail below. A more exact analysis of the effect of pressure can only be obtained through detailed numerical simulations.

The effect of MTZ on specific productivity is to reduce the effective length of bed which also translates to a reduction in working capacity. Again, this can be represented generally as some function $f_{2}$ of the MTZ. The specific productivity can therefore be written as

specific productivity $=\frac{k\left[W C-f_{1}(\Delta P)-f_{2}(M T Z)\right]}{\tau}$

where $f_{1}$ and $f_{2}$ are functions of $\Delta P$ and MTZ, respectively. Since the cycle time is inversely proportional to bed velocity

$\tau \propto \frac{1}{U}$

The specific productivity equation can be further rearranged to specific productivity

$$
\begin{aligned}
& =k^{\prime}\left[W C-f_{1}(\Delta P)-f_{2}(M T Z)\right] U \\
& =k^{\prime}\left[W C-f_{1}(\Delta P(U))-f_{2}(M T Z(U))\right] U
\end{aligned}
$$

where $k^{\prime}$ is another proportionality constant and the dependence of pressure drop and mass transfer zone on velocity is made explicit. In order to find $f_{1}$ and $f_{2}$, we can consider a linear isotherm. For no pressure drop in the system, the ideal amount of adsorbate loading per mass of adsorbent is given by

$W C=\Delta n_{\text {ideal }}=K\left(P_{H}-P_{L}\right)$

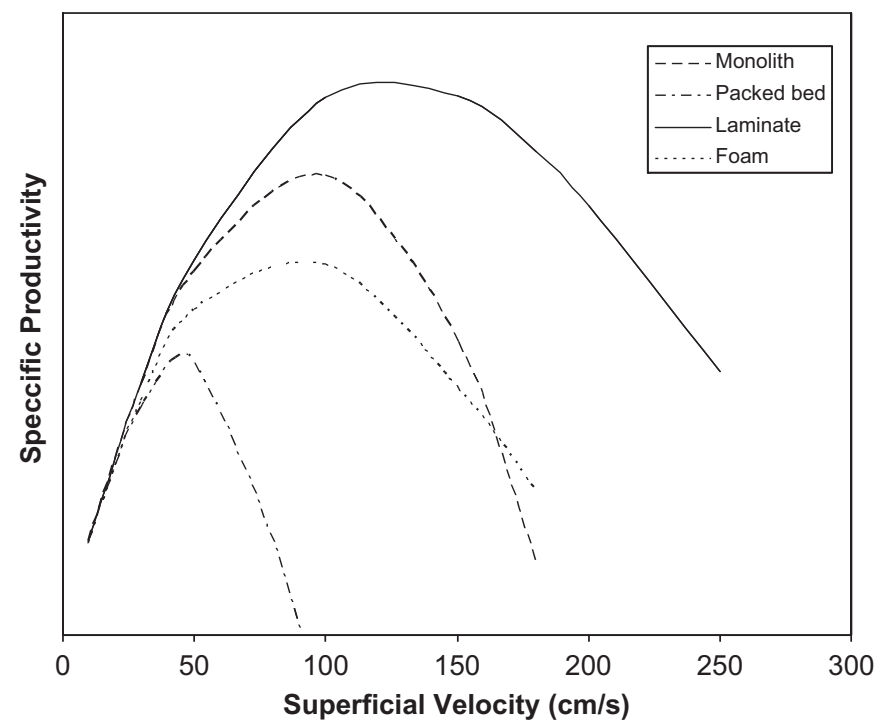

Fig. 10. Comparison of specific productivity for pellet, laminate, foam and monolith as a function of superficial gas velocity.

As mentioned earlier, pressure drop affects the process by reducing the average pressure swing between feed and blowdown in PSA system. The working capacity for the system with pressure drop and a mass transfer zone is therefore (to a first approximation)

$$
\begin{aligned}
\Delta n_{\text {non-ideal }} & =\left(K\left(\left(P_{H}-\frac{\Delta P}{2}\right)-\left(P_{L}+\frac{\Delta P}{2}\right)\right)\right)\left(1-\frac{M T Z}{L}\right) \\
& =\left(K\left(P_{H}-P_{L}\right)-K \Delta P\right)\left(1-\frac{M T Z}{L}\right) \\
\Delta n_{\text {non-ideal }} & =\left(\Delta n_{\text {ideal }}-K \Delta P\right)\left(1-\frac{M T Z}{L}\right)
\end{aligned}
$$

Therefore, the specific productivity of the adsorbent system can be represented by following correlation:

specific productivity $=k^{\prime}\left(\Delta n_{\text {ideal }}-K \Delta P\right)\left(1-\frac{M T Z}{L}\right) U$

Eq. (38) indicates that specific productivity is proportional to the superficial velocity and decreases with increase in $M T Z$ and pressure drop. Therefore any factor affecting the MTZ and pressure drop may influence the specific productivity. Specifically, the trade-off in specific productivity as a function of velocity is clear. At high velocities, cycle time is reduced, however, the pressure drop and MTZ length increase which decrease overall system performance. At low velocities, pressure drop and MTZ effects are small but the long cycle time lead to low specific productivity.

Fig. 10 shows qualitatively the specific productivity as a function of superficial velocity for different structures as given by Eq. (38). $\Delta n_{\text {ideal }}, K$ and $\Delta P$ values were calculated based on isotherm data. It can be seen that although at lower velocities, the productivity is almost the same for different structures since all dispersive effects are negligible, however, at higher velocities, the structured adsorbents display better performance than the conventional packed bed of pellets. It is precisely this higher velocity region that structured adsorbents are most suited to and display their superiority. Comparison of the optimal velocity shows that a laminate is capable of almost twice the specific productivity of a packed bed of pellets. To convert the $y$-axis in Fig. 10 to a quantitative scale will require detailed simulations for the specific system of interest. The intention 
here, however, is to illustrate the qualitative trends expected in performance as a function of throughput.

\section{Conclusion}

In this study, some important features of different adsorbent structures were derived and their performance compared. Mathematical models were developed based on structural and geometric parameters that impact overall system performance such as voidage, external surface area per unit volume and bulk density. Limiting LDF type expressions were derived for laminates, monoliths and foam structures. Pressure drop and mass transfer characteristics were investigated. Based on the simulation results, laminate systems were shown to be potentially very promising structures provided they can be made with small spacings ( $<0.2 \mathrm{~mm}$ ) and sheet widths $(0.2 \mathrm{~mm})$. Monoliths can be considered as successful candidates if appropriate cell densities ( $>1000 \mathrm{cpsi}$ ) and voidages are used. The actual values to use depend on the effective diffusivities that can be obtained for diffusion in the monolith walls. Low density monoliths are penalized not only by having less mass per unit volume, but also by having far less external surface area per unit volume than a packed bed. The overall rate constant is, therefore, diminished considerably compared to a packed bed. As shown theoretically, the contribution of axial dispersion to overall rate constant was trivial for almost all adsorbent configurations, while the internal rate and external film contributions were, respectively, about $75 \%$ and $25 \%$. To compensate for reduced mass, a faster cycle is needed; however, the interstitial velocity rapidly rises and stretches the mass transfer zone, so that it may be impossible to match a packed bed performance with a low density monolith. As we progress in monolith development, it will be important to attempt to lower the voidage and increase the cell density. Foam structures on the other hand show comparable properties to packed beds.

Finally, we show that the throughput for alternate adsorbent structures may be substantially higher than a packed bed provided the structure is operated at its optimal velocity. Increasing beyond this value leads to an increase in pressure drop and mass transfer zone which more than offsets the gains in cycle time reduction.

\section{Notation}

specific surface area per unit bed volume, $\mathrm{cm}^{2} / \mathrm{cm}^{3}$ bulk concentration, $\mathrm{g} \mathrm{mol} / \mathrm{cm}^{3}$ average solid concentration, $\mathrm{g} \mathrm{mol} / \mathrm{cm}^{3}$ gas concentration in voids in adsorbent, $\mathrm{g} \mathrm{mol} / \mathrm{cm}^{3}$ gas concentration at adsorbent surface, $\mathrm{g} \mathrm{mol} / \mathrm{cm}^{3}$ effective diffusivity, $\mathrm{cm}^{2} / \mathrm{s}$ axial dispersion coefficient, $\mathrm{cm}^{2} / \mathrm{s}$ molecular diffusion coefficient, $\mathrm{cm}^{2} / \mathrm{s}$ equation constant parameter total mass transfer coefficient, $\mathrm{cm} / \mathrm{s}$ equation constant parameter film mass transfer coefficients, $\mathrm{cm} / \mathrm{s}$ pore mass transfer coefficients, $\mathrm{cm} / \mathrm{s}$ column length, $\mathrm{cm}$ molar (radial) flux of component, $\mathrm{g} \mathrm{mol} / \mathrm{cm}^{2} \mathrm{~s}$ molar flux at adsorbent surface, $\mathrm{g} \mathrm{mol} / \mathrm{cm}^{2} \mathrm{~s}$ average adsorbent loading, g mol $/ \mathrm{kg}$ adsorbent adsorbent loading, $\mathrm{g} \mathrm{mol} / \mathrm{kg}$ zeolite inner wall radii of monolith channel, $\mathrm{cm}$ outer wall radii of monolith channel, $\mathrm{cm}$ superficial fluid velocity, $\mathrm{cm} / \mathrm{s}$ wall thickness, $\mathrm{cm}$

\section{Greek letters}

$\alpha \quad$ geometry's factor, $\mathrm{cm}^{-1}$

$\varepsilon_{b} \quad$ bed void fraction

$\varepsilon_{p} \quad$ solid void fraction

$\rho_{s} \quad$ adsorbent density (includes binder, support, etc.), $\mathrm{g} / \mathrm{cm}^{3}$

\section{Acknowledgement}

The authors would like to acknowledge the Monash Research Graduate School (MRGS) for providing scholarship support for Fateme Rezaei.

\section{Appendix A. Model for monoliths}

\section{A.1. Gas phase equations}

Single-component adsorption is considered. The monolith is represented by a series of parallel cylinders. We solve for the breakthrough in one cylinder only since all the cylinders are the same (assume no maldistribution). A differential mass balance for the adsorbate in the gas phase flowing through the bed gives

$$
\left(\frac{\partial C}{\partial t}\right)_{z}-\frac{D_{L}}{\varepsilon_{b}} \frac{\partial^{2} C}{\partial z^{2}}+\frac{1}{\varepsilon_{b}} \frac{\partial(U C)}{\partial z}+\frac{a k_{f}}{\varepsilon_{b}}\left(C-C_{s}\right)=0
$$

The specific surface area for the monolith per unit volume can be represented as

$a=\frac{2}{R_{1}}$

\section{A.2. Solid phase equations}

A differential mass balance for the adsorbate inside the monolith wall is given by

$\varepsilon_{p}\left(\frac{\partial C^{p}}{\partial t}\right)_{r}+\rho_{s} w\left(\frac{\partial q^{p}}{\partial t}\right)_{r}+\frac{1}{r}\left(\frac{\partial(r N)}{\partial r}\right)_{t}=0$

The volume-mean concentration is given by

$\bar{C}^{p}=\frac{1}{\pi\left(R_{2}^{2}-R_{1}^{2}\right)} \int_{R_{1}}^{R_{2}} 2 \pi C^{p} d r=\frac{2}{R_{2}^{2}-R_{1}^{2}} \int_{R_{1}}^{R_{2}} r C^{p} d r$

$\bar{q}^{p}=\frac{2}{R_{2}^{2}-R_{1}^{2}} \int_{R_{1}}^{R_{2}} r q^{p} d r$

Considering the boundary condition of $\left.\frac{\partial C}{\partial r}\right|_{r=R_{2}}=0$ and substituting the above equations into the differential equation gives

$\varepsilon_{p}\left(\frac{\partial \bar{C}^{p}}{\partial t}\right)+\rho_{s} w\left(\frac{\partial \bar{q}^{p}}{\partial t}\right)+\frac{2 R_{1}}{R_{2}^{2}-R_{1}^{2}} N_{s}=0$

In this equation, $N_{S}$ is the flux at $R=R_{1}$ which is given by

$N=k_{f}\left(C-C_{s}\right)$

In terms of an overall driving force this can also be written as

$N_{s}=k_{p}\left(C_{s}-\bar{C}^{p}\right)$

In order to specify $N_{s}$, continuity at the pellet surface is considered:

$k_{f}\left(C-C_{S}\right)=-D_{e}\left(\frac{\partial C^{p}}{\partial r}\right)_{R_{1}}$ 
In this equation, $D_{e}$ is the "effective diffusion coefficient" for the adsorbate in the adsorbent wall. To apply this equation, the concentration profile within the adsorbent is approximated by a parabola:

$C^{p}=A(t)+B(t) r^{2}$

Considering $C^{p}=C_{S}$ at $r=R_{1}$, it follows that

$\left(\frac{\partial C^{p}}{\partial r}\right)_{R_{1}}=\frac{4 R_{1}}{R_{2}^{2}-R_{1}^{2}}\left(C_{S}-\bar{C}^{p}\right)$

Finally, the surface flux and concentration can be solved in terms of $C$ and $\bar{C}_{1}^{p}$ as

$N=\frac{k_{f} k_{p}}{k_{f}+k_{p}}\left(C-\bar{C}^{p}\right)$

$N=\bar{k}\left(C-\bar{C}^{p}\right)$

$\frac{1}{\bar{k}}=\frac{1}{k_{f}}+\frac{1}{k_{p}}$

Considering $k_{p}=4 D_{e} R_{1} / R_{2}^{2}-R_{1}^{2}$, the overall mass transfer coefficient is given by

$\bar{k}=\frac{1}{\frac{1}{k_{f}}+\frac{R_{2}^{2}-R_{1}^{2}}{4 R_{1} D_{e}}}$

Assuming constant velocity and linear isotherm $(q=K C)$, the gas and solid phase conservation equations can be summarized as

$$
\begin{aligned}
& \left(\frac{\partial C}{\partial t}\right)_{z}-\frac{D_{L}}{\varepsilon_{b}} \frac{\partial^{2} C}{\partial z^{2}}+\frac{U}{\varepsilon_{b}} \frac{\partial C}{\partial z}+\frac{a \bar{k}}{\varepsilon_{b}}\left(C-\bar{C}^{p}\right)=0 \\
& \left(\frac{\partial \bar{C}^{p}}{\partial t}\right)_{z}=\frac{2 \varepsilon_{p} R_{1} \bar{k}\left(C-\bar{C}^{p}\right)}{\left(R_{2}^{2}-R_{1}^{2}\right)\left(\varepsilon_{p}+\rho_{s} w K\right)}
\end{aligned}
$$

\section{References}

Ackley, M.W., Smolarek, J., Leavitt, F.W., 2003. Pressure swing adsorption gas separation method, using adsorbents with high intrinsic diffusivity and low pressure ratios, United States Patent and Trademark Office Granted US6506234.

Brandani, F., Rouse, A., Brandani, S., Ruthven, D.M., 2004. Adsorption kinetics and dynamic behavior of a carbon monolith. Adsorption 10 (2), 99-109.

Cybulski, A., Moulijn, J.A., 1998. Structured Catalysts and Reactors, p. 1

Ergun, S., 1952. Fluid flow through packed columns. Chemical Engineering Progress 48 (2), 89-94.

Gadkaree, K.P., 1997. System and method for adsorbing contaminants and regenerating the adsorber, United States Patent and Trademark Office Granted US5658372.

Giani, L., Groppi, G., Tronconi, E., 2005. Mass-transfer characterization of metallic foams as supports for structured catalysts. Industrial and Engineering Chemistry Research 44 (14), 4993-5002.

Gibson, L.J., Ashby, M.F., 1997. Cellular Solids: Structure and Properties. Cambridge University Press, Cambridge.
Golden, T.C., Golden, C.M.A., Zwilling, D.P., 2003. Self-supporting absorbent fabric for gas separation, United States Patent and Trademark Office Granted US6565627.

Grande, C.A., Cavenati, S., Barcia, P., Hammer, J., Fritz, H.G., Rodrigues, A.E., 2006. Adsorption of propane and propylene in zeolite $4 \mathrm{~A}$ honeycomb monolith Chemical Engineering Science 61 (10), 3053-3063.

Groppi, G., Giani, L., Tronconi, E., 2007. Generalized correlation for gas/solid masstransfer coefficients in metallic and ceramic foams. Industrial and Engineering Chemistry Research 46 (12), 3955-3958.

Hawthorn, R.D., 1974. Afterburner catalysts-effects of heat and mass transfer between gas and catalyst surface. A.I.Ch.E. Symposium 70 (137), 428-438.

Hyun, S.H., Danner, R.P., 1982. Equilibrium adsorption of ethane, ethylene, isobutane, $\mathrm{CO}_{2}$, and their binary mixtures on $13 \mathrm{X}$ molecular sieves. Journal of Chemical Engineering Data 27, 196-200.

Keefer, B.G., Carel, A., Sellars, B., Shaw, I., Larisch B., 2004. Adsorbent laminate structures, United States Patent and Trademark Office Granted US6692626.

Kodama, A., Goto, M., Hirose, T., Kuma, T., 1993. Experimental study of optimal operation for a honeycomb adsorber operated with thermal swing. Journal of Chemical Engineering of Japan 26 (5), 530-535.

Lee, L.Y., Perera, S.P., Crittenden, B.D., Kolaczkowski, S.T., 2000. Manufacture and characterisation of silicalite monoliths. Adsorption Science and Technology 18 (2), 147-170.

Li, Y.Y., Perera, S.P., Crittenden, B.D., 1998. Zeolite monoliths for air separation, part 2: Oxygen enrichment, pressure drop and pressurization. Trans, p. 76.

Maurer, R.T., 1994. Spiral-wound adsorber module, United States Patent and Trademark Office Granted US5338450.

Patcas, F.C., Garrido, G.I., Kraushaar-Czarnetzki, B., 2007. CO oxidation over structured carriers: a comparison of ceramic foams, honeycombs and beads. Chemical Engineering Science 62 (15), 3984-3990.

Patton, A., Crittenden, B.D., Perera, S.P., 2004. Use of the linear driving force approximation to guide the design of monolithic adsorbents. Chemical Engineering Research and Design 82 (8), 999-1009.

Rezaei, F., Webley, P.A., 2009. Application of structured adsorbents in gas separation Processes-a review. Separation Science and Technology, in review.

Richardson, J.T., Peng, Y., Remue, D., 2000. Properties of ceramic foam catalyst supports: pressure drop. Applied Catalysis A: General 204 (1), 19-32.

Richardson, J.T., Remue, D., Hung, J.K., 2003. Properties of ceramic foam catalyst supports: mass and heat transfer. Applied Catalysis A: General 250 (2), 319-329.

Rode, E.J., Boulet, A.J.J., Pelman, A.M., Babicki, M.L., Keefer, B., Sawada, J.A., AlizadehKhiavi, S., Roy, S., Gibbs, A.C., Kuznicki, S.M., 2007. Engineered adsorbent structures for kinetic separation, World Intellectual Property Organization Patent W0200660743432.

Ruthven, D.M., 1984. Principles of Adsorption and Adsorption Processes. Wiley, New York.

Ruthven, D.M., Thaeron, C., 1996. Performance of a parallel passage adsorbent contactor. Gas Separation and Purification 10 (1), 63-73.

Sawad, J.A., Alizadeh-Khiavi, S., Roy, S., Kuznicki, S.M., 2005. High density adsorbent structures, United States Patent and Trademark Office Granted W02005032694.

Sircar, S., Kumar, R., Anselmo, K.J., 1983. Effects of column nonisothermality or nonadiabaticity on the adsorption breakthrough curves. Industrial and Engineering Chemistry Process Design and Development 22 (1), 10-15.

Taylor, G.I., 1954. Diffusion and mass transport in tubes. Proceedings of the Physica Society Section B 67 (12), 857-869.

Wakao, N., Funazkri, T., 1978. Effect of fluid dispersion coefficients on particle-tofluid mass transfer coefficients in packed beds: correlation of sherwood numbers Chemical Engineering Science 33 (10), 1375-1384.

Yates, M., Blanco, J., Martin-Luengo, M.A., Martin, M.P., 2003. Vapour adsorption capacity of controlled porosity honeycomb monoliths. Microporous and Mesoporous Materials 65 (2-3), 219-231.

Young, L.C., Finlayson, B.A., 1974. Mathematical modeling of the monolith converter Advances in Chemistry Series (13), 629-643.

Yu, F.D., Luo, L.A., Grevillot, G., 2002. Adsorption isotherms of VOCs onto an activated carbon monolith: experimental measurement and correlation with different models. Journal of Chemical and Engineering Data 47 (3), 467-473.

Zabka, M., Minceva, M., Rodrigues, A.E., 2006. Experimental and modeling study of adsorption in preparative monolithic silica column. Chemical Engineering and Processing: Process Intensification 45 (2), 150-160.

Zabka, M., Gomes, P.S., Rodrigues, A.E., 2008. Performance of SMB with conventional and monolith columns. Separation Purification Technology 63 (2), 324-333. 